\title{
Correspondence
}

\section{Cannabinoids for postoperative analgesia?}

To the Editor:

We read with interest the article on the effects of nabilone on postoperative pain by Pierre Beaulieu. ${ }^{1}$ While it is unfortunate that his results did not generate firm conclusions, we believe that descriptive subgroup analyses may have yielded some important information regarding the effects of nabilone on postoperative morphine consumption and side-effects. Nineteen of the 41 patients in the study underwent hysterectomy or myomectomy, which is a surgically homogenous patient population for whom it would have been interesting to have compared the effects of placebo, ketoprofen and nabilone doses of $\mathrm{l}$ and $2 \mathrm{mg}$ on morphine consumption, visual analogue scale (VAS) scores and secondary outcomes. Eighteen of the 41 patients underwent orthopedic surgery (hip or knee arthroplasty), which represents a different surgical population of potentially greater heterogeneity, with an additional confounding factor that knee arthroplasties are associated with more postoperative pain compared with hip arthroplasty. ${ }^{2}$ A greater number of knee arthroplasty patients in the nabilone $2 \mathrm{mg}$ group could explain why there is an apparent possible hyperalgesic effect of nabilone. Hence, it would be interesting to know the breakdown of knee/hip arthroplasty by study group (placebo, ketoprofen and nabilone 1 and $2 \mathrm{mg}$ ), and the results (morphine consumption, VAS scores and secondary outcomes) according to the orthopedic procedure and study group.

Étienne de Médicis MD MSC FRCPC

Jean-Pierre Tétrault MD MSC FRCPC

Centre hospitalier universitaire de Sherbrooke, Sherbrooke, Canada

E-mail: estrial@globetrotter.net

Accepted for publication November 6, 2006.

\section{References}

1 Beaulieu $P$. Effects of nabilone, a synthetic cannabinoid, on postoperative pain. Can J Anesth 2006; 53: 769-75.

2 Pang WW, Hsu TC, Tung CC, Hung CP, Chang DP, Huang $M H$. Is total knee replacement more painful than total hip replacement? Acta Anaesthesiol Sin 2000; 38: 143-8.

\section{Reply:}

Thank you to Dr. de Médicis and Dr. Tétrault for their interesting comments. While it is true that recruited patients in the referenced study underwent primarily gynecological and orthopedic procedures, the patients were distributed into four different groups, making it difficult to perform appropriate sub-group analysis with the limited number of subjects in each group.

The suggestion that more patients in the nabilone 2-mg group underwent knee arthroplasty is in fact, correct. Nevertheless, if excluding the three patients who underwent knee arthroplasty, the amount of morphine patient-controlled analgesia used in $24 \mathrm{hr}$ (the primary outcome variable) in the nabilone 2-mg group was higher than previously reported (average of $49.5 \mathrm{mg}$ compared to $45.4 \mathrm{mg}$, with no significant differences between the four groups). Furthermore, the intensity of pain at rest (24 hr), which was significantly different between groups, remained so, even if knee arthroplasty patients were excluded from the analysis. In contrast, when considering the pain scores on movement (24 hr), there were no statistical differences, although mean pain scores in the nabilone 2-mg group remained numerically greater, and only decreased from 7.7 to $7.1(P=0.09)$. Therefore, despite the fact that more patients in the nabilone 2-mg group underwent knee arthroplasty, this fact did not markedly influence pain scores which remained highest in this group, despite the similarity of morphine consumption in the two groups.

Pierre Beaulieu MD PhD

CHUM - Hôtel-Dieu, Montreal, Canada

E-mail: pierre.beaulieu@umontreal.ca

\section{Angioedema, angiotensin converting enzyme inhibitors, and angiotensin receptor blocking drugs}

To the Editor:

We read the review by Sarkar et al. ${ }^{1}$ with great interest. Although angioedema can cause rapid, progressive airway compromise, facial and oral cavity edema as a complication of angiotensin converting enzyme 


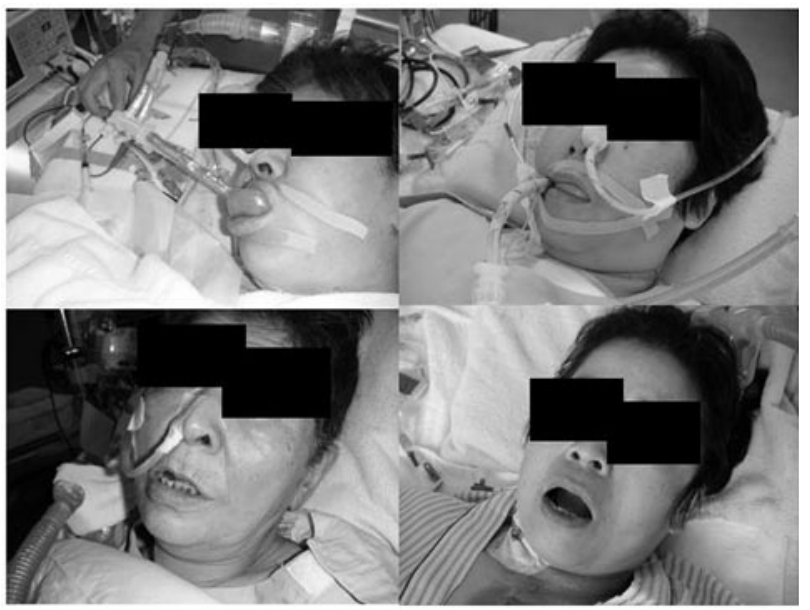

FIGURE The pictures in the upper panels show the extent of tongue angioedema in two patients immediately after cardiopulmonary bypass surgery. The lower panels demonstrate complete resolution in both patients two days postoperatively, with conservative management.

inhibitors (ACEIs) therapy is under-recognized, as described in the review article. ${ }^{1}$ A potential role of angiotensin receptor blocking (ARB) drugs should also be considered.

Recently, we experienced two cases strongly suspected of being secondary to angioedema, following cardiopulmonary bypass $(\mathrm{CPB})$ surgery. Both patients had obvious tongue swelling immediately after their operations (Figure, upper panels). We initially suspected impaired venous return or traumatic sequelae resulting from the endotracheal tube or bite block as potential etiologies. However, the tongues of both patients were pink-coloured, without evidence of venous congestion, thus making an obstructive circulatory disturbance unlikely. Eyelid edema was not observed, and fluid balance and serum protein and albumin concentrations were within normal ranges. Heart failure and increases in central venous pressure were also excluded as possible causes in the setting of pulmonary catheter measurements that were within accepted physiological ranges.

On further review, it was identified that the first patient was being treated for hypertension with candesartan, an ARB drug, and the second patient had been receiving long-term therapy with enalapril, an ACEI. As with all ACEIs, enalapril potentiates the effects and augments the survival of bradykinin in humans. ${ }^{2}$ Preoperative ACEIs augment the kinin response to $\mathrm{CPB}^{3}$ and increased serum bradykinin levels have been reported in association with $\mathrm{CPB} .{ }^{4}$
Theoretically, ARBs do not increase bradykinin levels and the complication might not be induced by ARBs. However, angioedema associated with ARBs in a patient who had experienced angioedema secondary to ACEIs has been described. ${ }^{5}$ Patients receiving not only ACEIs but also ARBs ${ }^{6}$ might be susceptible to angioedema, and $\mathrm{CPB}$ could present a precipitating factor. Other triggering factors, such as recent airway intervention, trauma, or exposure to irritant fumes have also been discussed. ${ }^{1}$ We suggest that cardiac surgery with $\mathrm{CPB}$ might present an additional risk factor for angioedema in patients receiving ACEIs, ARBs, or a combination of these two drugs.

Although tongue swelling disappeared within a few hours in both patients with conservative management (Figure, lower panels), we monitored them carefully for several days to ensure airway patency. Considering the increasingly common use of ACEIs and ARBs for the treatment of hypertension and heart failure, anesthesiologists should consider these drug-related factors as a potential cause of angioedema in the perioperative setting, especially for cardiac surgery patients.

Yushi U. Adachi MD PhD

Satoko Iwakiri MD

Takasumi Katoh MD PhD

Hamamatsu University School of Medicine,

Hamamatsu, Japan

E-mail: yuadachi@hama-med.ac.jp

Accepted for publication November 10, 2006.

\section{References}

1 Sarkar P, Nicholson G, Hall G. Brief review: angiotensin converting enzyme inhibitors and angioedema: anesthetic implications. Can J Anesth 2006; 53: 9941003.

2 Bonner G, Preis S, Schunk U, Toussaint C, Kaufmann $W$. Hemodynamic effects of bradykinin on systemic and pulmonary circulation in healthy and hypertensive humans. J Cardiovasc Pharmacol 1990; 15(suppl. 6): S46-56.

3 Pretorius M, McFarlane JA, Vaughan DE, Brown NJ, Murphey LJ. Angiotensin-converting enzyme inhibition and smoking potentiate the kinin response to cardiopulmonary bypass. Clin Pharmacol Ther 2004; 76: 379-87.

4 Cugno M, Nussberger J, Biglioli P, Alamanni F, Coppola $R$, Agostoni A. Increase of bradykinin in plasma of patients undergoing cardiopulmonary bypass: the importance of lung exclusion. Chest 2001; 120: 177682.

5 Abdi R, Dong VM, Lee CJ, Ntoso KA. Angiotensin II receptor blocker-associated angioedema: on the heels 
of ACE inhibitor angioedema. Pharmacotherapy 2002; 22: 1173-5.

6 Irons BK, Kumar A. Valsartan-induced angioedema. Ann Pharmacother 2003; 37: 1024-7.

\section{Reply:}

Thank you for the opportunity of replying to the letter of Dr. Adachi and colleagues.

Dr. Adachi and colleagues documented two cases of tongue edema in patients following cardiopulmonary bypass. Following exclusion of other possible precipitating causes, it was concluded that angioedema secondary to angiotensin converting enzyme inhibitor (ACEI) therapy was the most likely diagnosis. These two cases highlight some of the more interesting aspects of angioedema secondary to ACEI therapy discussed in our review.

Firstly, use of ACEI medication is increasing and clinicians will be faced with the complications of therapy. It is therefore important that they are aware of this potentially life threatening complication. Secondly, although theoretically angiotensin receptor blocker (ARB) drugs should not cause angioedema, these case reports emphasize that ARBs cannot be considered completely safe. ${ }^{1-3}$ Finally, it is unfortunate that there are no definitive symptoms or investigations to confirm the diagnosis which is based on a high index of suspicion and exclusion of other possible causes of angioedema. Clinical awareness of this potentially life threatening complication and prompt intervention remain the keys to successful management.

Grainne Nicholson MB BS

George Hall PhD

St George's Hospital Medical School, London, UK E-mail: gnichols@sgul.ac.uk

\section{References}

1 van Rijnsoever EW, Kwee-Zuiderwijk WJ, Feenstra J. Angioneurotic edema attributed to the use of losartan. Arch Intern Med 1998; 158: 2063-5.

2 Chin AG, Krowiak EJ, Deeb ZE. Angioedema associated with angiotensin II receptor antagonists: challenging our knowledge of angioedema and its etiology. Laryngoscope 2001; 111: 1729-31.

3 Howes $L G$, Tran D. Can angiotensin receptor antagonists be used safely in patients with previous ACE inhibitorinduced angioedema? Drug Saf 2002; 25: 73-6.

\section{Training pathways for perioperative transesophageal echocardiography}

To the Editor:

We write in regards to the just-published Canadian guidelines for perioperative transesophageal echocardiography (TEE), ${ }^{1}$ and the accompanying editorial. ${ }^{2}$ We first of all wish to commend the group for creating the guidelines at all; that a clearly written document, supported by evidence where available, was produced by such a large number of authors is testimony to the effort and thought that went into the document. However, the recommendation that the basic level of proficiency be attained in a minimum of three months dedicated TEE training, with all studies completed within a year, is at odds with the understanding that adult learning be needs-based and individualized. The authors cite references supporting the minimum number of studies required, but they do not provide evidence for the timelines suggested, and for many centres reality will dictate that the basic level of proficiency will be achieved by people who will either combine clinical work with their practical TEE training, or will not provide the service at all.

The editorial states that the guidelines "extend the expected proficiency of an individual trained at the basic level to include limited diagnostic abilities [in recognition of] the expertise one would realistically expect of a specialist physician who undertakes three months of dedicated training in TEE". However, the guidelines indicate that any abnormalities found require consultation with an advanced level practitioner if treatment will be significantly influenced. The reasoning behind the more demanding requirements for basic training then becomes circular. Put another way, if proficiency in TEE is thought of as a continuum from beginner, through basic, to advanced skills, perhaps the point at which the authoring group has elected to place the "basic skills" label is at the wrong point along the skill spectrum: far enough to make it difficult for any but academic centres to train practitioners, but not far enough to make them independent.

All but one of our present TEE practitioners created their own learning program, and most of the handson training was done locally. It requires considerable effort on the trainee's part, not to mention a great deal of support from our colleagues in anesthesiology and cardiology who have advanced skills, to develop one's abilities in this way. It is disappointing to have this route of training ignored by the guidelines, and the ramifications not addressed in the editorial. We 